\title{
Performance analysis of Robust WDM fast circuit-switched networks with token passing in control channel
}

\author{
Samir A. Abd-Elmalak, Chintan Vaishnav and Anura P. Jayasumana*,† \\ Department of Electrical and Computer Engineering, Colorado State University, Fort Collins, CO 80523, U.S.A.
}

\begin{abstract}
SUMMARY
WDM networks depend on predetermined, fixed wavelength channels, requiring highly stable lasers and tunable filters. Robust WDM networks, based on dynamically selected wavelengths, relax manufacturing and operating wavelength tolerance requirements leading to low-cost implementations. A node dynamically selects a currently unused wavelength for communication, and uses it for a limited period of time for communication with a destination, thus allowing the network to tolerate wavelength variation with time. A performance model is presented for Robust WDM networks in which the right to establish WDM connections is arbitrated periodically by a control channel based on token passing. Comparison with simulation results indicates the model's ability to predict the network performance accurately. Copyright (C) 2002 John Wiley \& Sons, Ltd.
\end{abstract}

KEY WORDS: WDM; optical networks; Robust WDM; medium-access protocols

\section{INTRODUCTION}

One way to exploit the massive bandwidth of the optical fibre is to use concurrent multiple node-to-node transmissions. Wavelength-division multiple access (WDMA) [1-5] relies on concurrent connections on different wavelengths and is thus able to relieve the electronic bottleneck. The minimum spacing between adjacent channels can be reduced by using stable transmitters (lasers) that do not drift too far from their nominal operating wavelength range. The present implementations of WDMA networks are based on transmitters and receivers that can be precisely tuned to predetermined fixed wavelengths. Wavelength of lasers drift with temperature and the output power of the laser can also change even though a constant input current is applied. Typical temperature dependent drifts of distributed feedback (DFB) and vertical-cavity surface-emitting lasers (VCSELs) are of the order of $0.1 \mathrm{~nm} /{ }^{\circ} \mathrm{C}$, whereas Fabry-

\footnotetext{
*Correspondence to: Anura P. Jayasumana, Department of Electrical and Computer Engineering, Colorado State University, Fort Collins, CO 80523, U.S.A.

†E-mail: Anura.jayasumana@colostate.edu

Contract/grant sponsor: National Science Foundation; contract/grant number ECS9412944

Contract/grant sponsor: DOD Advanced Research Projects Agency, Micro Electronics Technology Office
}

Published online 28 January 2002

Copyright (C) 2002 John Wiley \& Sons, Ltd.
Received 15 March 2001

Revised 18 September 2001 Accepted 21 September 2001 
Perot (FP) lasers exhibit $0.5 \mathrm{~nm} /{ }^{\circ} \mathrm{C}$ drifts. For a wavelength drift of $0.1 \mathrm{~nm} /{ }^{\circ} \mathrm{C}$ and an operating range of $20-50^{\circ} \mathrm{C}$, the spacing between adjacent channels has to increase by $3 \mathrm{~nm}$, in addition to that due to other considerations. This necessitates the temperature of the components to be tightly stabilized. Wavelength and power stabilization of laser transmitters is thus critical and contributes to a significant increase in cost. Thus, commercial exploitation of WDM systems has not made great progress in short-haul local-area network (LAN) environment where the cost of the fibre and its installation would be considerably low compared to the cost of suitable precise and stabilized transmitter sources. Also, the cost of using stabilized, i.e. thermally isolated, DFB lasers is about one order of magnitude higher than that of non-isolated ones.

Robust WDM network is an architecture that relaxes manufacturing and operating wavelength tolerances [6-13] and as a result reduces the cost to levels acceptable for LAN environments. A demonstration prototype has been implemented and several protocols have been proposed for Robust WDM networks [14-17]. Robust WDM networks use an access protocol that can adapt to large variations of wavelengths of lasers over both limited and extended periods of time. The medium-access control (MAC) protocol does not depend on fixed wavelength channels, but dynamically adapts to the variations of the signal wavelengths. Thus, a Robust WDM network does not require extremely stable lasers or tunable filters and can be implemented with low-cost optical components, thus leading to cost-effective network implementations.

Robust WDM networks depend on a control channel-based MAC protocol that ensures orderly and fair establishment of connections. The protocol used in the control channel can have a significant effect on the performance. In this paper, we present a performance model for a symmetric fast circuit-switched Robust WDM network. In Section 2, the architecture and the MAC protocol are explained. The performance model is developed in Section 3. The model is evaluated in Section 4 for different network parameters and the results are compared with these generated by a simulator.

\section{ROBUST WDM NETWORKS}

\subsection{Architecture}

We describe the Robust WDM architecture and the receiver only to the extent that is necessary to develop the performance model. A detailed description of the Robust WDM concepts, architecture and receiver can be found in References [9,10,15-17].

The Robust WDM network architecture is based on a passive star as shown in Figure 1. Each node has an array of lasers, one of which is dynamically selected at the transmission time. The time is divided into slots, each of which consists of two intervals, namely the transmission interval $\left(T_{\mathrm{t}}\right)$ and the reservation interval $\left(T_{\mathrm{R}}\right)$ as shown in Figure 2. High-speed WDM transmissions are allowed only during the transmission intervals. Using WDM, several stations, up to the total number of channels $(c)$, may transmit during a transmission interval, as long as they are on different channels (wavelengths). During a reservation interval, all the stations have to cease high-speed WDM transmissions. A waiting station, which is assigned to use that reservation interval, finds an available channel (if one exists) corresponding to a wavelength that is not currently used by any other station. Only the station that acquires the reservation interval can select an available channel, even if there is more than one channel available and there is 


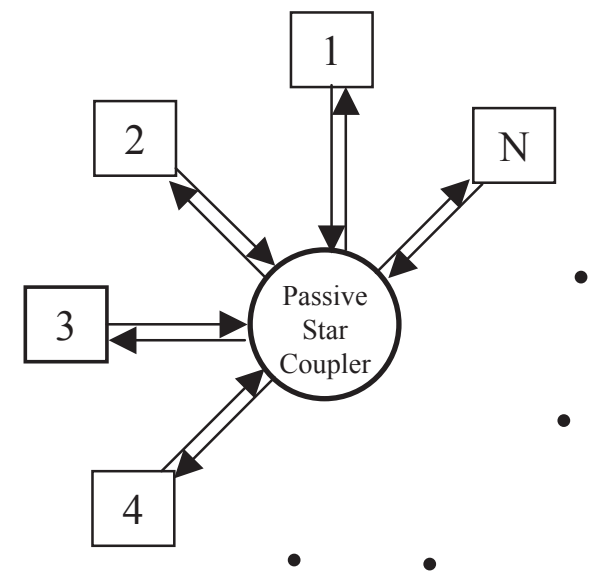

Figure 1. WDM network using broadcast star topology.

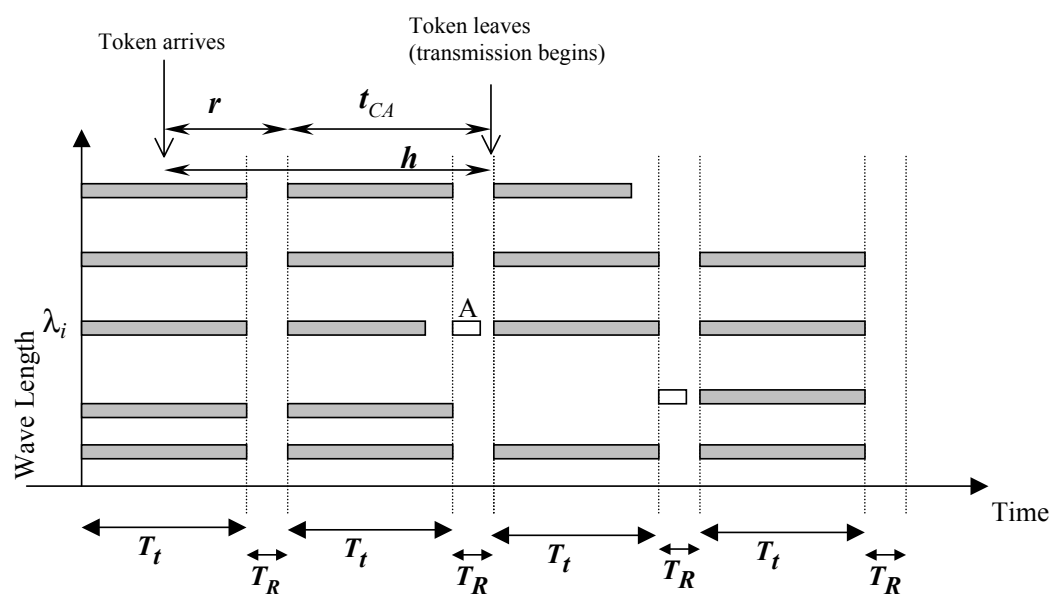

Figure 2. The interlaced WDM transmission intervals $\left(T_{\mathrm{T}}\right)$ and reservation intervals $\left(T_{\mathrm{R}}\right)$.

more than one station requiring a channel at that moment. Once an unused wavelength is found, the station establishes a link with the intended destination to be used at the beginning of the next transmission interval. Each reservation interval is followed by a transmission interval in which all the WDM links resume their operation and the new link starts its transmission as well. The reservation interval may also be used to acknowledge whether or not the receiver was able to lock-on to the signal as well as to establish a reverse link if needed. The allocation of the reservation intervals among stations is done using a control channel. In this paper, we consider the case where the arbitration is carried out using token passing in this control channel. 


\subsection{Wavelength-tolerant tracking receiver}

Robust WDM architecture depends on the wavelength-tolerant tracking receiver shown in Figure 3. A detailed discussion of its hardware and a successful introductory demonstration can be found in References [16-18]. The optical power incoming from lasers that operate at different wavelengths is spectrally decomposed by the dispersion system, which can be realized by a blazed grating or a planar-guided wave dispersion system. The decomposed optical signals are imaged onto an over-sampled array of photodetectors that convert photonic data into electronic signals and pass them to a preamplifier stage and, subsequently, to a winner-take-all (WTA) decoder through the field-effect transistor (FET) switches. Note that the position of the detector detecting the incoming signal has a one to one relationship with its wavelength. The WTA decoder consists of a high-speed integrated flash controller that converts the position of the incoming electrical signals into amplitude values and finds very rapidly the detector element(s) at the brightest wavelength. Since we allow only one station to transmit during a reservation interval, the WTA decoder at each of the stations can quickly lock onto the wavelength being transmitted. The WTA decoder finds out the spatial pin-diode detector position corresponding to the transmitting laser and sends this information to the selector/router. The selector/router is basically a programmable crossbar-switching network that gets its routing information from a wavelength translation table. During the multi-wavelength data transmission phase, only the current from a single detector element or from a few neighbouring PINs corresponding to a narrow wavelength band being received by the particular station, identified during a prior reservation interval is decoded and sent to the quantizer.

During an initialization phase for the network, all the lasers are switched on, one after another, and each station in the system creates and stores its own translation table in its selector/ router for the wavelength-PIN conversation. In a network operation environment, the receiver(s) will be switched periodically into this calibration mode and, hence, wavelength drifts of lasers can be tracked by updating the wavelength translation table(s). This procedure assumes that the time scale of laser drifting is larger than that of the data transmission. A network controller ties the transmitter and receiver, as well as carries out the medium-access control functions [18].

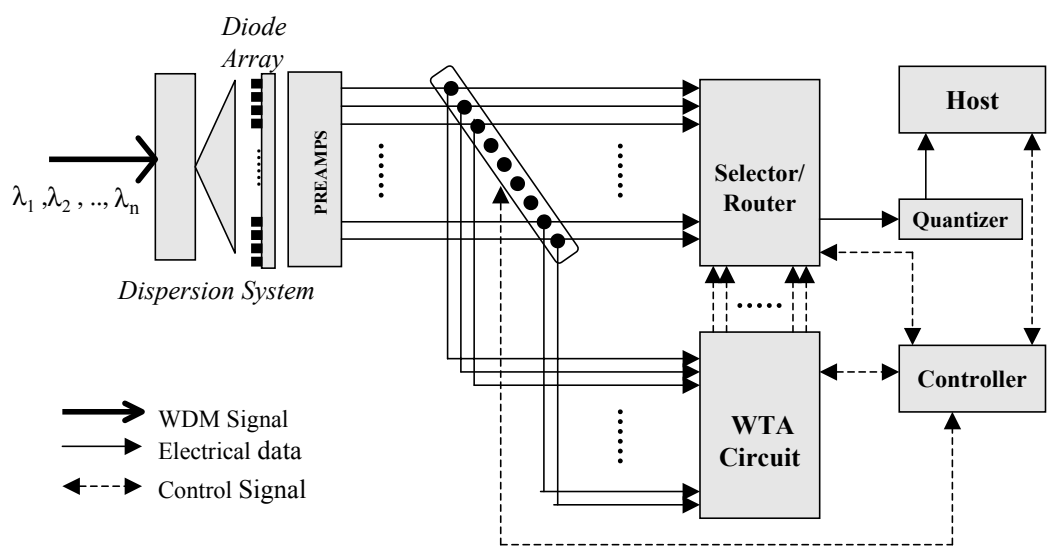

Figure 3. Robust WDM Receiver. 
Although the use of such a reservation interval, during which no WDM transmissions are allowed, decreases the efficiency of the network, this protocol and the implementation possess many advantages over the traditional WDM techniques in the areas of robustness and cost effectiveness. The reasoning for having a reservation interval and how it enhances robustness is addressed elsewhere [16,17]. Note that, in this context, the term available channel refers to a wavelength that is not used by any other station, rather than a predefined wavelength. Thus, the spacing between adjacent channels may vary and need not be constant. The protocol is able to tolerate slow variation of the laser wavelength, as a node can be forced to re-select another laser after transmitting for a certain limited time period. This time period need to be short enough to ensure that the wavelength will not drift into an adjacent channel during the transmission. The variation of laser wavelengths with time is however not considered in our model and a fixed number of channels is assumed. The protocol analysed here is one of many that can be used in wavelength tolerant LANs [9].

\subsection{Token-based MAC protocol}

The medium access protocol aims at regulating the network access to provide fair access to all the stations on the network. The MAC scheme has to ensure that only one station uses any given reservation interval. In this paper, we consider a network, where a token, passed on a control channel, visits the stations in a cyclic order and determines the one which would access a given reservation interval. Only a station that has a connection request and is waiting to establish a connection can hold the token. Upon receiving the token, the station keeps it until the intended connection is established. Only the station holding the token is permitted to establish a new connection during a reservation interval. Stations with no connection requests or those involved in on going transmissions do not hold the token, but pass it to the next downstream station.

Only an idle station that has a request for a connection can hold the token until it gets an available channel, corresponding to a wavelength that is not used by any other station (e.g. $\lambda_{i}$ in Figure 2). Once such a wavelength is found, the station establishes a connection with its desired destination. The reservation interval may also be used to acknowledge whether or not the receiver was able to lock-on to the signal as well as to establish a reverse link if needed. After getting an available channel, the station is allowed to start its data transmission at the beginning of the next transmission interval. A station is considered 'active' if it starts or continues its WDM transmission in the current slot; otherwise, it is said to be 'idle'. A station is 'full' if it has a connection request that is incomplete, i.e. it is pending or being served. An 'empty' station does not have any requests. A station that gets the token while it is active or empty passes it immediately to the downstream station.

To illustrate the link establishment procedure, consider the scenario illustrated in Figure 4. Having received a connection request from its host, node A wishes to establish a connection with its intended destination (station B). Station waits until it is visited by the token, which is the license to initiate a reservation interval. For performance considerations, the reservation intervals have to be separated by minimum time duration (minimum inter-reservation time). After ensuring the elapse of this minimum duration, station A broadcasts a stop transmission signal, ST(s), on the control channel, denoted by s. The stop transmission signal includes the source address (station A), the destination address (station B), and the type of connection (simplex or full duplex) to be established. Upon receiving this signal, all the stations cease their 


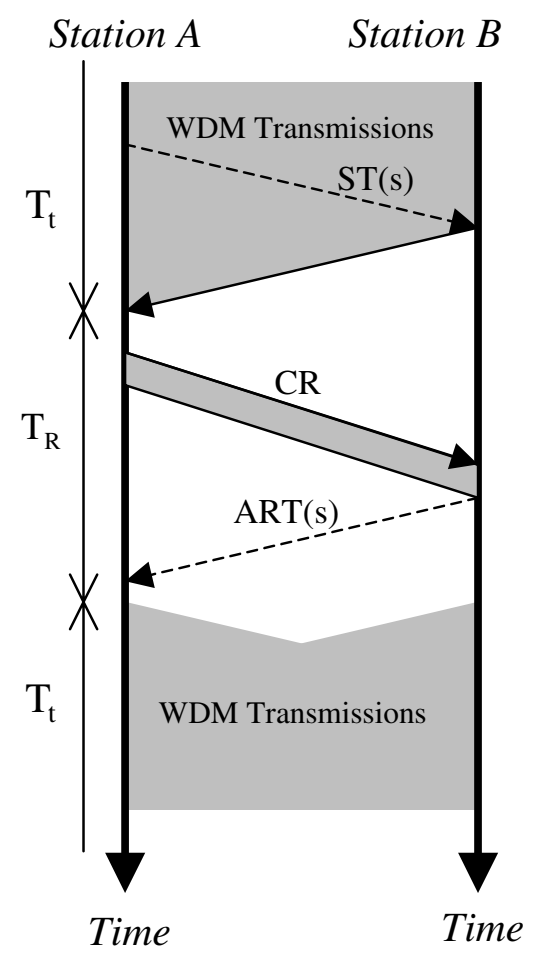

Figure 4. Successful reservation interval for a simplex connection from A to B.

on-going high-speed WDM transmissions, marking the beginning of the reservation interval. Station A selects one of its lasers that operates on a wavelength in the currently unused portion of the spectrum, as measured by its receiver's PIN position. Once such a wavelength is found and while there is no other power on the fibre, station A uses that wavelength to send a connection request (CR) to inform all stations including $\mathrm{B}$ which wavelength it will use. Recognizing itself as the destination, station B checks the type of connection (simplex or full duplex). If a simplex connection is required, station B sends an acknowledgment to station A followed by a acknowledge and resume transmission, ART(s), signal to all the stations on the control channel. In the case of a full-duplex connection, station B sends the acknowledgment and then follows the same procedure to establish a reverse link. Upon receiving the connection request, station $\mathrm{A}$ sends an acknowledgment to station $\mathrm{B}$ followed by a resume transmission signal to all stations on the control channel. Upon detecting the resume transmission signal, the reservation interval ends and the transmission interval begins, i.e. all stations resume their WDM transmissions, so does A (and B in duplex mode) using the new WDM links established between them. This marks the end of the reservation interval and the beginning of the transmission interval.

Figure 4 illustrate scenarios of a successful reservation interval (i.e. one that results in successful connection establishment); however, two cases of blocking may be expected. First, channel blocking occurs when the source node (station A) fails to find any of its lasers operating 
in an unused part of the wavelength spectrum. Second, receiver blocking occurs if the destination node (station B) for the connection request is also a destination for an ongoing WDM transmission. Channel and receiver blocking would lead to immediate aborting of the reservation interval with the broadcast of the resume transmission signal by stations $\mathrm{A}$ and $\mathrm{B}$, respectively. Blocking due to signaling errors is not considered in the analysis below, as the probability of such an error is very small [7]. Similar reservation-based protocols employing a control channel have been presented in Reference [9] where the waiting stations contend during the reservation interval to get a channel.

\section{THE MODEL}

In this section, we develop an analytical model for a circuit-switched Robust WDM network that consists of $N$ stations and $c$ transmission channels. Each station is assumed to have sufficient number of lasers to cover all the channels. A symmetric network is considered with respect to the arrival rates and the service time distributions at the nodes. The process of connection request arrivals to an empty station is assumed to be Poisson with mean $\lambda_{s}$ arrivals per sec per station. A station can handle only one connection request at a time; thus, no arrivals occur at a station that has an incomplete circuit-switched connection request. The service times of connections are assumed to be independent and independent of the arrival process. The service time is geometrically distributed, with parameter $p$ and mean length $L$ slots. The token transmission times $(E)$, from a station to another, is constant. Only simplex WDM transmissions are considered although the model can easily be extended to cover the duplex case. This analysis also assumes that the reservation interval $T_{\mathrm{R}}$ and the transmission interval $T_{\mathrm{t}}$ are fixed. A more general version of a protocol can be conceived where the duration of these two intervals are not fixed, however, in such a case the added hardware complexity has to be taken into account.

The token is passed from station to station in the signaling channel. As shown in Figure 5 if a station, visited by the token, has a connection request (say $i$ ) that has not been served yet, it holds the token (for time $h_{i}$,) until it gets a WDM channel, before sending the token to the next station. This corresponds to waiting for a reservation interval in which it can find a channel. In the example of Figure 2, the node has to wait for a second reservation interval at which time it can use a wavelength $\lambda_{i}$. The WDM transmission lasts for $x_{i}$ time units and the pattern repeats. The token can therefore be considered as a server that serves a station for a time $h_{i}$, as shown in Figure 5.

Since the token serves a station for only one request at a time, it can be considered as serve-atmost-one discipline. Assuming constant token transmission times $(E)$, the waiting time $\left(W_{Q}\right)$ can be stated using the results given in References $[19,20]$ as follows:

$$
W_{Q}=\frac{(1-\rho)}{(1-\rho-E \lambda)}\left[\frac{(N-1) E}{2(1-\rho)}+\frac{\lambda H^{(2)}}{2(1-\rho)}+\frac{E}{2}+H\right]
$$

where, $W_{Q}$ is the mean total waiting time, i.e. the interval between the connection request arrival and beginning of the corresponding transmission, $H, H^{(2)}$ the first and second moments of the token service time $h$ (visit period of the token), $\lambda$ the total arrival rate to the network [arrivals/s], and $\rho$ is the total token utilization. 


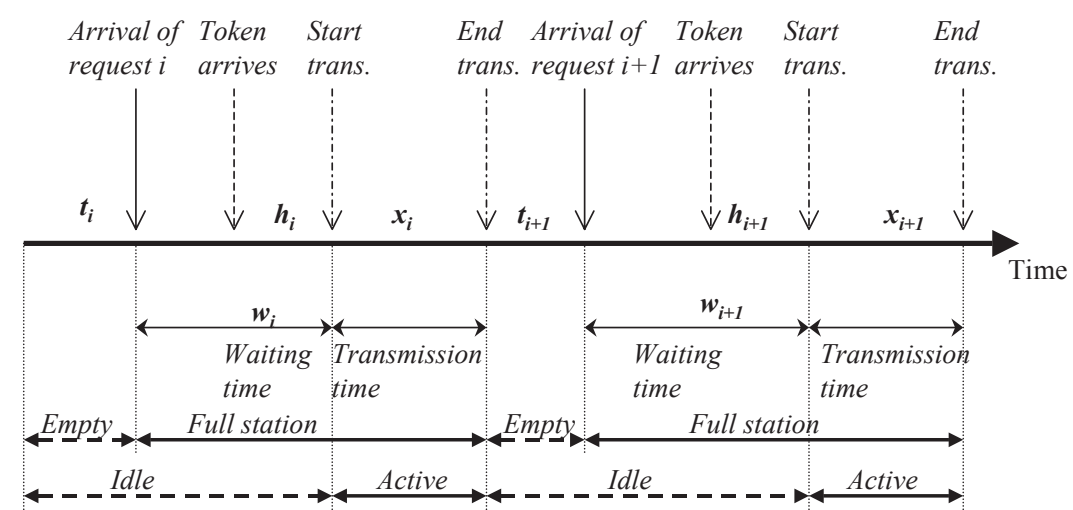

Figure 5. Events taking place at a station and the corresponding state of the station.

Also,

$$
\lambda=N \lambda_{s} P_{\mathrm{ES}}
$$

where, $P_{\mathrm{ES}}$ is the probability that a given station is empty, thus

$$
\rho=\lambda H
$$

Referring to Figure 5, let $w_{i}$ be the waiting time of connection request arrival $i ; E\left[w_{i}\right]=W_{Q}, t_{i}$ the time between the end of transmission of request $(i-1)$ and the instant of next $(i$ th) connection request's arrival. $E\left[t_{i}\right]=1 / \lambda_{s}$, assuming a Poisson arrival process, $h_{i}$ the token holding time until the WDM connection of request $i$ is established. $E\left[h_{i}\right]=H$, and $x_{i}$ be the transmission time of connection request $i ; E\left[x_{i}\right]=X$.

In Figure 5, the station is initially empty (ES). Since the arrival process is Poisson, the mean time until the next request arrival is $\left(1 / \lambda_{s}\right)$; and only then does the station become full (FS). The arrived message waits for a random time with a mean value $W_{Q}$ before its transmission starts. The transmission time has a mean value $X$. A station is considered 'full,' i.e. in state (FS), from the instant of receiving a connection request till the end of its transmission. A station without such a request is considered an empty station, i.e. in state ES. Thus,

$$
P_{\mathrm{ES}}=1 /\left[1+\lambda_{s}\left(W_{Q}+X\right)\right]
$$

Substituting values of $\lambda, \rho$ and $P_{\mathrm{ES}}$ from Equations (2)-(4) into Equation (1) and rearranging, we get

$$
A\left(W_{Q}\right)^{2}+B W_{Q}+D=0
$$

where

$$
\begin{gathered}
A=2 \lambda_{s} \\
B=2+[2 X-2(N+1) H-3 N E] \lambda_{s} \\
D=-\left\{N E+2 H+\left[(X-H) N E+N H^{(2)}+2 H(X-N H)\right] \lambda_{s}\right\}
\end{gathered}
$$


The above equation for $W_{Q}$ can be used to evaluate the waiting time, provided $H$ and $H^{(2)}$ are known.

Evaluation of $H$ and $H^{(2)}$ :

$H$ and $H^{(2)}$ can be evaluated, from Figure 2, as follows:

$$
\begin{gathered}
H=E\left[r+t_{C A}\right]=R+T_{C A} \\
H^{(2)}=E\left[\left(r+t_{C A}\right)^{2}\right]=R^{(2)}+2 R T_{C A}+\left(T_{C A}\right)^{(2)}
\end{gathered}
$$

where

$$
\begin{gathered}
T_{C A}=\sum_{i=0}^{\infty} i S P_{C A}(i) \\
T_{C A}^{(2)}=\sum_{i=0}^{\infty}(i S)^{2} P_{C A}(i)
\end{gathered}
$$

Derivation details of these equations can be found in Reference [7]. $R, R^{(2)}$ correspond to the first and second moments of the residual time $(r)$, where $r$ is the time interval between the arrival instant of token at a station till the beginning of the next transmission interval as shown in Figure 2.

$T_{C A},\left(T_{C A}\right)^{(2)}$ are the first and second moments of $t_{C A}$, the time from the beginning of a transmission interval to the time at which the token leaves the station as in Figure 2. The slot time $S$ is given by

$$
S=\left(T_{\mathrm{t}}+T_{\mathrm{R}}\right)
$$

Let

$$
\begin{aligned}
P_{C A}(i)= & \operatorname{Prob}\{\text { there is a channel available exactly } i \text { slots } \\
& \text { after receiving the token and not before that }\} \\
= & P(E \cap F) \quad=P(E \mid F) P(F)
\end{aligned}
$$

where $E$ and $F$ denote the first and second clauses in the above definition.

Assuming that the duration of a connection is geometrically distributed with a mean value $L$ slots/connection,

$$
\begin{aligned}
q & =\operatorname{Prob}\{\text { at the beginning of a slot, the transmitting station under consideration } \\
& \quad \text { gives up the channel, i.e. the station becomes 'idle' }\} \\
& =1 /(L+1)
\end{aligned}
$$

Let $p=1-q$.

Assume simplex WDM transmissions and let $(t=0)$ correspond to the beginning instant of the transmission interval next to the token arrival, where $t$ is time expressed in slots.

Next we evaluate $P_{C A}(i)$. First consider the case where $N>c$ :

$P(E \mid F)=$ Prob \{exactly $i(>0)$ slots after receiving the token,

a station or more of the $c$-active stations become(s) idle $\mid$ all the

$c$-active stations are active for $i$-successive slots from $t=0$ \}

According to the geometric distribution assumption, an active station may keep active or become idle in the next slot with probability $(p)$ and $(1-p)$, respectively, independent of how 
long it has been active. Thus,

$$
\begin{aligned}
P(E \mid F)= & \operatorname{Prob}\{\text { a station or more of the } c \text {-active stations become(s) idle } \mid \text { there were } \\
& =1-p^{c}
\end{aligned}
$$

Regarding $P(E \mid F)$, the $c$-active stations at $t=0$ may include a new one that starts right at that moment. To exclude this situation, we have to be sure that there were $c$-active stations at $t=-1$ (i.e. in the previous slot) that keep active (continue) for another slot. Hence,

$$
\begin{aligned}
& P(F)= \operatorname{Prob}\{\text { there are } c \text {-active stations at } t=-1 \text { and all of them keep active for } \\
&(i+1) \text {-successive slots }\} \\
&= \text { Prob }\{\text { all the } c \text {-active stations keep active for }(i+1) \text {-successive slots } \mid \text { there } \\
&\quad \text { are } c \text {-active stations at } t=-1\} . \operatorname{Prob}\{\text { there are } c \text {-active stations at } t=-1\} \\
&=p^{c(i+1)} P_{C S A}
\end{aligned}
$$

Hence, for $N>c$ and $i>0$ :

$$
P_{C A}(i)=\left(1-p^{c}\right) p^{c(i+1)} P_{C S A}
$$

Substituting Equation (10) into Equations (8) and (9), we get

$$
\begin{gathered}
T_{C A}=\left[b^{2} /(1-b)\right] S P_{C S A} \\
\left(T_{C A}\right)^{(2)}=\left[b^{2}(1+b) /(1-b)^{2}\right] S^{2} P_{C S A}
\end{gathered}
$$

where $b=p^{c}$, and

$$
P_{C S A}=\text { Prob }\{\text { there are } c \text {-active stations at the beginning of a transmission interval }\}
$$

Thus, Equations (11), (12), (6) and (7) can be used to find $H$ and $H^{(2)}$ provided $P_{C S A}$ is known.

Note that for $N \leqslant c$, which corresponds to a network in which there are more channels than nodes,

$$
\begin{gathered}
P_{C A}(i)=1 \quad(\text { for } i=0) \\
P_{C A}(i)=0 \quad \text { (otherwise). }
\end{gathered}
$$

Using this in Equations (8) and (9), to evaluate $T_{C A}$ and $T_{C A}^{(2)}$, and using Equations (6) and (7), we get the following, for $N \leqslant c$ :

$$
H=R \quad \text { and } \quad H^{(2)}=R^{(2)}
$$

Evaluating $P_{C S A}$ :

To find $P_{C S A}$, the discrete-time Markov chain, shown in Figure 6, is used. The state is defined as the number of active stations $(n)$ at the beginning of a transmission interval. The state $n=c$ represents the case where all the $c$-channels of the network are busy; i.e. no channel is available. If there is a channel available, the only station that is allowed to start its transmission is the one that is holding the token. Thus, the number of the active stations can increase by at most one. On the other hand, an arbitrary number of active stations may complete their transmissions in the same time slot and hence the state of the chain can decrease by any number. 


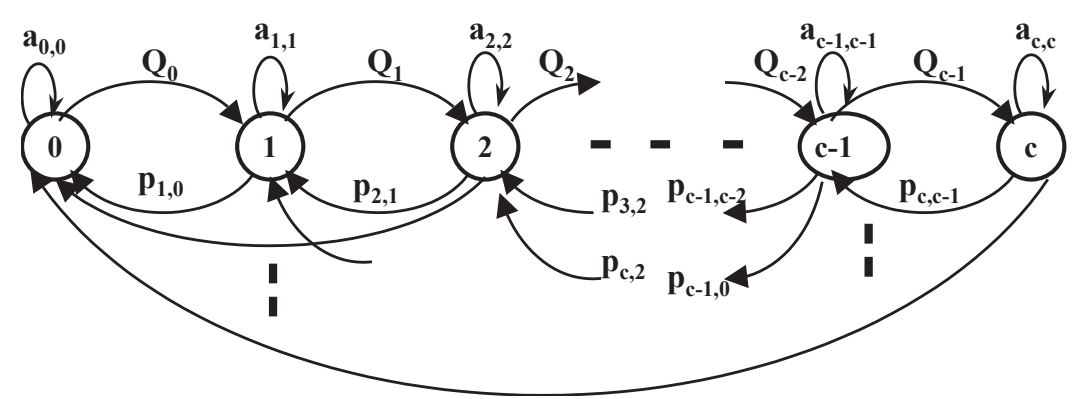

Figure 6. Markov chain for the number of active stations.

The steady-state probabilities can be obtained by solving Equations (14) and (15) below. Thus $P_{C S A}$, which corresponds to being in state $c$ in the Markov chain, can be obtained.

$$
\begin{gathered}
\pi=\pi P \\
\sum_{j=0}^{c} p_{j}=1
\end{gathered}
$$

where

$$
\pi=\left[\begin{array}{lllll}
p_{0} & p_{1} & p_{2} & \cdots & p_{c}
\end{array}\right]
$$

$p_{j}=$ Prob $\{$ having $j$ active stations at the beginning of a transmission interval $\}$ $P=\left[p_{i, j}\right]$ is the matrix of the transition probabilities $(i, j=0,1,2, \ldots, c)$.

Its elements are shown below, and the non-zero elements are calculated as follows:

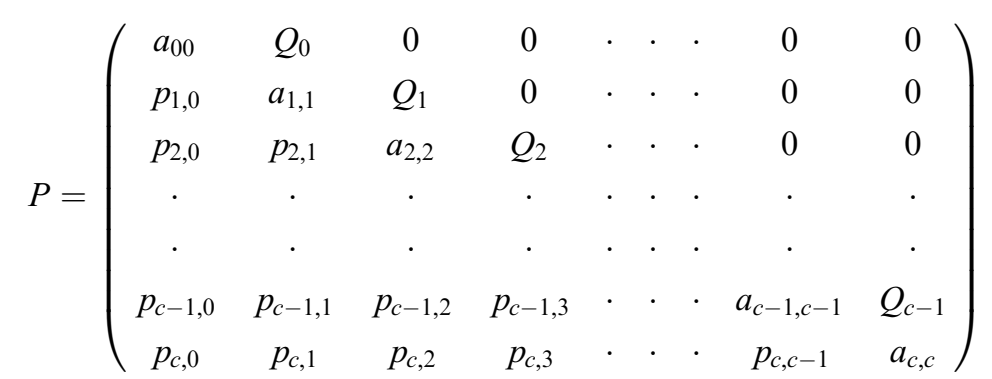

$p_{n, m}=$ Prob $\{$ the number of active stations in the current slot is $m \mid$ that number was $n$ in the previous slot\}

$=$ Prob \{out of the $n$-active stations, $m$ continue and the rest quit $\}$

- Prob \{the number of (idle and full) stations, out of the other $(N-n)$ stations $=0 \mid$ all the $(N-n)$ stations were idle during the previous slot $\}$ 
+ [ Prob \{out of the $n$-active stations, $(m-1)$ continue and the rest quit\}

- Prob \{the number of (idle and full) stations, out of the other $(N-n)$ stations

$\geqslant 1 \mid$ all the $(N-n)$ stations were idle during the previous slot $\}]$

$$
\begin{aligned}
p_{n, m} & =\left[{ }^{n} C_{m} p^{m} q^{(n-m)}\left(1-P_{S I F}\right)^{N-n}+{ }^{n} C_{(m-1)} p^{(m-1)} q^{(n-(m-1))}\left[1-\left(1-P_{S I F}\right)^{N-n}\right]\right] \text { for } m>0 \\
p_{n, m} & =q^{n}\left(1-P_{S I F}\right)^{N-n} \text { for } m=0 \\
P_{S I F} & =\text { Prob }\{\text { a station is full } \mid \text { it was idle in previous slot }\}
\end{aligned}
$$

Using the same arguments as above, $Q_{n}$ and $a_{n, n}$ can be obtained as follows.

$Q_{n}=$ Prob $\{$ the number of active stations in the current slot is increased

by one $(=n+1) \mid$ there were $n$-active stations in the previous slot\}

This can be shown to be the following, as given by Reference [7]:

$$
Q_{n}=p^{n}\left[1-\left(1-P_{S I F}\right)^{N-n}\right] \text { for } n>0
$$

$Q_{0}=$ Prob \{there is a station or more, from the $N$ idle stations, that had an arrival or more during the previous slot\}

$$
Q_{0}=1-E^{-N \lambda_{s} S}
$$

$a_{n, n}=$ Prob $\{$ the number of active stations $(n)$ in the current slot is the same as that inthe previous slot\}

It can be shown that [7],

$$
a_{n, n}=p^{n}\left(1-P_{S I F}\right)^{N-n}+n P^{n-1} q\left[1-\left(1-P_{S I F}\right)^{N-n}\right] \text { for } c>n>0
$$

Similarly,

$$
\begin{array}{ll}
a_{c, c} & =p^{c}+c p^{c-1} q\left[1-\left(1-P_{S I F}\right)^{N-c}\right] \quad \text { for } n=c \\
a_{0,0}=e^{-N \lambda_{s} S} &
\end{array}
$$

To evaluate $P_{S I F}$, consider only the idle interval in Figure 5, where the station is either empty or full with a waiting request; thus, $P_{S I F}$ can be obtained as follows:

$$
P_{S I F}=\lambda_{s} W_{Q} /\left[1+\lambda_{s} W_{Q}\right]
$$

Solving Equations (14) and (15), we get $P_{C S A}$, which is used in Equations (11) and (12) to get $T_{C A}$ and $\left(T_{C A}\right)^{(2)}$, respectively. Substituting in Equations (6) and (7) for $T_{C A}$ and $\left(T_{C A}\right)^{(2)}$, together with $R$ and $R^{(2)}$ [7], we get $H$ and $H^{(2)}$, respectively. Using these values as well as Equation (13) to calculate $A, B$ and $D$ and then solving Equation (5) and selecting the appropriate root, $W_{Q}$ is obtained.

\section{NUMERICAL EXAMPLES}

In the following analysis, we consider a network with following parameters: $N=50$ stations, $c=10$ channels, $T_{\mathrm{R}}=1 \mu \mathrm{s}, T_{\mathrm{t}}=100 \mu \mathrm{s}, L=100$ slots $/$ connection, $\lambda_{s}=10$ or 100 arrivals $/ \mathrm{s}$ and 


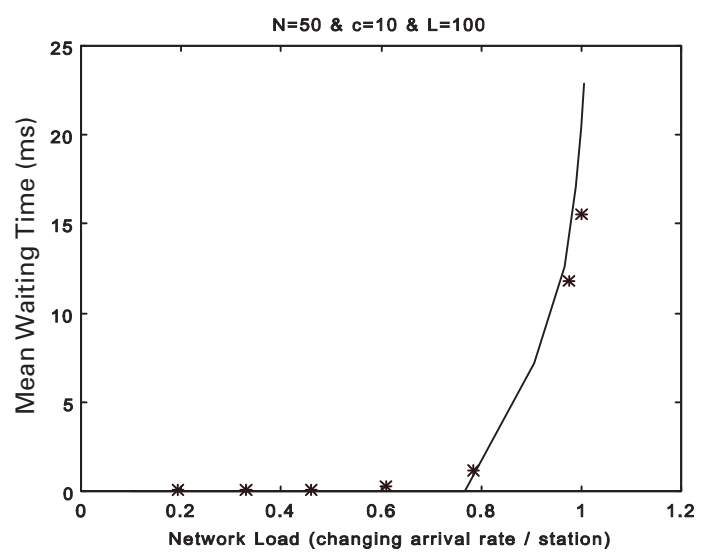

Figure 7. Mean waiting time $\left(W_{Q}\right)$ vs. network load $(G)$.

$E=0.1 \mu \mathrm{s}$. These values are kept fixed, unless otherwise mentioned. The network load $(G)$ is given by $G=\lambda X / c$, where $X=L\left(T_{\mathrm{t}}+T_{\mathrm{R}}\right)$. Note that even though the arrival rate per station is kept constant, the accepted load to the network $G$ changes due to the unity buffer limitation at each node.

Solving Equation (5), we get two values for the mean arrival waiting time $\left(W_{Q}\right)$. In all experiments, the two values of $W_{Q}$ are either one is positive and one is negative or both are positive. In the former case, the positive one is considered. In case of two positive roots, both values are used to ensure that each of $P_{\mathrm{ES}}$ and $\rho$ are less than or equal to one. Always, only one feasible solution exists.

To illustrate the effect of the different parameters on $W_{Q}$, one parameter is changed at a time, keeping the others constant. Results obtained by using the model described in this paper are compared with those generated by simulation [21]. In all the following figures the analytic results are plotted with solid lines; while the simulation results are represented by asteriks.

Figure 7 illustrates the variations of $W_{Q}$ with offered load $G$. Changing $G$ is achieved by varying the arrival rate at each station, $\lambda_{s}$. At low arrival rates $\left(\lambda_{s}\right)$, increasing $\lambda_{s}$ decreases $P_{\mathrm{ES}}$, as in Equation (4), and hence increases the number of full stations. Thus the token rotation time, the time taken by the token to circulate once among all the stations, increases which increases $W_{Q}$. As $\lambda_{s}$ increases further, the network saturates in the sense that all stations are full almost all the time, and hence limiting $G$. Upon reaching the upper limit of $G$, increasing $\lambda_{s}$, does not increase $\lambda$ and thus limits $W_{Q}$ to its maximum value.

In Figure 8, the relation between $W_{Q}$ and $c$ is shown. In general, increasing $c$, decreases the mean token holding time $(H)$, and hence decreases $W_{Q}$. In this case, the traffic admitted to the network, which is defined as $G$ above, varies between 0.3 and 5.05. The variations of the mean waiting time $\left(W_{Q}\right)$ with the number of slots per connection $(L)$, is shown in Figure 9. The mean duration of a connection $(X)$, in seconds, is given by $X=L S$, where $\left(S=T_{\mathrm{t}}+T_{\mathrm{R}}\right)$ is the slot duration. For small values of $L$, in our case $\leqslant 200$, where the number of total arrivals during $X$ is $\leqslant c$, there is always a channel available. Thus, an arrival waits for no longer than the residual time $(r)$.

Increasing $L$ beyond that increases $X$ and hence increases the channel holding time by the transmitting station. As a result, the station holding the token waits for longer time $(H)$, until it gets an available channel. This in turn, increases the mean arrival waiting time $\left(W_{Q}\right)$. 


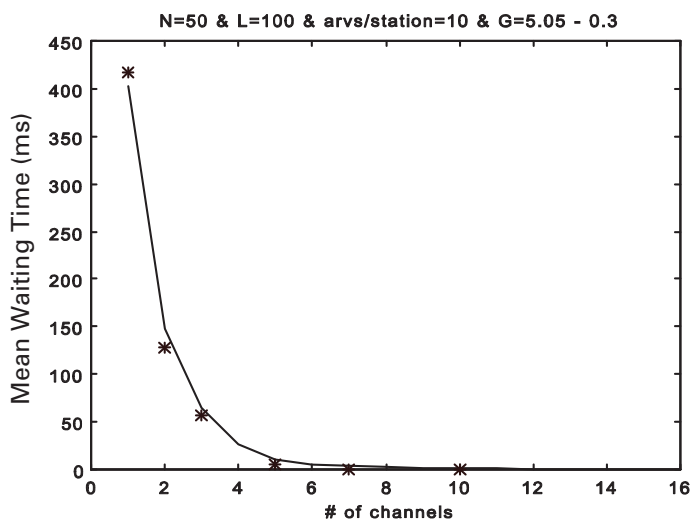

Figure 8. Mean waiting time $\left(W_{Q}\right)$ vs. number of channels $(c)$.

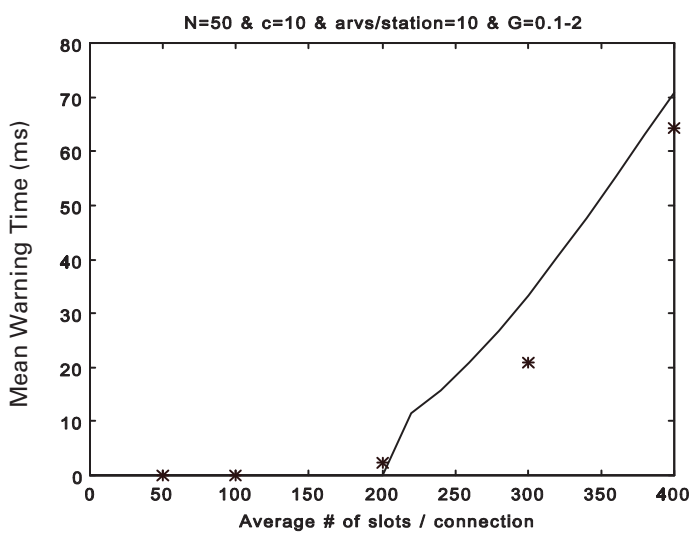

Figure 9. Mean waiting time $\left(W_{Q}\right)$ vs. connection duration $(X)$.

Figure 10 illustrates the changes of $W_{Q}$ with $N$. For $N \leqslant c$, there is always a channel available and hence an arrival waits for no longer than the residual time (r). After that, increasing $N$ increases the number of stations that may have an unserved request and thus holds the token. Hence, a station waiting time for the token $(T)$ increases and as a result $W_{Q}$ increases. Increasing $N$ further increases $T$ to a value that is long enough to let all other stations use the token to handle new requests waiting for the token. Thus, the token essentially serves all stations in a cyclic order. Hence, increasing $N$ increases the token rotation time; and as a result, the mean waiting time $\left(W_{Q}\right)$ increases almost linearly with $N$. $W_{Q}$ also almost linearly increases with $T_{\mathrm{R}}$, the duration of the reservation interval.

\section{CONCLUSION}

A symmetric Robust WDM circuit-switched network was modelled and analysed. In the model, the following assumptions are used: a fixed set of available channels, with each station having 


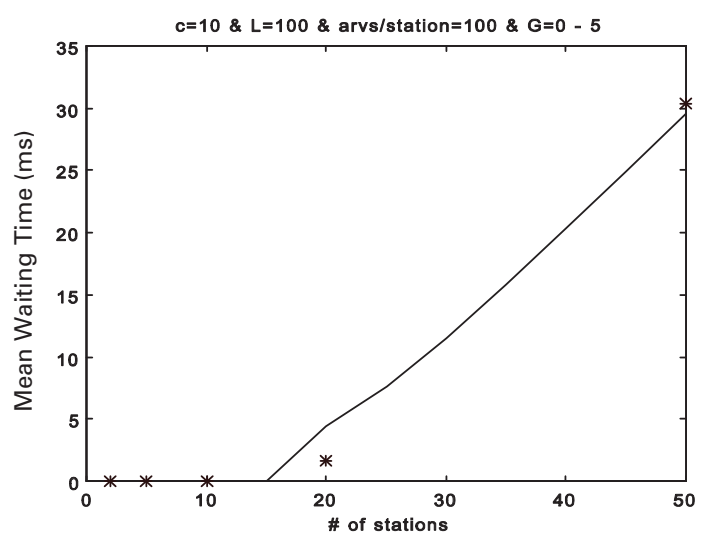

Figure 10. Mean waiting time $\left(W_{Q}\right)$ vs. number of nodes $(N)$.

enough lasers to cover all channels, one buffer per station that holds only one arrival, a symmetric network, Poisson arrivals, constant token transmission times, simplex WDM transmission and geometrically distributed WDM transmission times. The network uses a token-passing scheme on a separate low-speed control channel to assign one of the waiting stations the next reservation interval, during which that station seeks an available channel. Once the station finds an available channel, it establishes a circuit-switched connection with its intended destination. The effect of the different parameters on the mean waiting time $\left(W_{Q}\right)$ was investigated and results compared with simulation. The comparison indicates that the model can accurately predict the network's performance.

\section{ACKNOWLEDGEMENTS}

The authors acknowledge the feedback provided by Drs. J. Sauer, H. Temkin and T. El-Bawab for this work. This work was supported in part by the National Science Foundation under Grant No. ECS9412944, and a Grant from the DOD Advanced Research Projects Agency (ARPA), Micro Electronics Technology Office (MTO).

\section{REFERENCES}

1. Green Jr PE. Fibre Optic Networks. Prentice-Hall: Engelwood Cliffs, NJ, 1993.

2. Janniello FJ, Ramaswami R, Steinberg DG. A prototype circuit-switched multi-wavelength optical metropolitanarea network. IEEE Journal of Lightwave Technology 1993; 11(5-6):777-782.

3. Kazovsky LG, Poggiolini PT. STARNET: a multi-gigabit-per-second optical LAN utilizing a passive WD. Star. IEEE Journal of Lightwave Technology 1993; 11(5-6):1009-1027.

4. Mukherjee B. WDM-based local lightwave networks Part I: single-hop systems. IEEE Network 1992; 6(3):12-26.

5. Mukherjee B. WDM-based local lightwave networks Part II: multi-hop systems. IEEE Network 1992; 6(4):20-32.

6. Abd-Elmalak SA, Vaishnav C, Jayasumana AP. Performance of a packet switched WDM network with dynamic wavelength selection. Proceedings of IEEE 20th Local Computer Networking (LCN) Conference, October 16-19, Minneapolis, MN, 1995; 73-81.

7. Abd-Elmalak SA. Wavelength tolerant WDM networks: protocols, analysis and performance. Ph.D. Thesis, Department of Electrical Engineering, Colorado State University, 1997.

8. Abd-Elmalak SA, Vaishnav C, Jayasumana AP. Performance of a symmetric robust WDM network when the channel access pattern at nodes is known. Proceedings of IEEE 22nd Local Computer Networking (LCN) Conference, Minneapolis, MN, November 1997. 
9. El-Bawab TS, Vaishnav C, Jayasumana AP, Temkin H, Sauer J, Willebrand H. Medium access control protocols for robust wavelength division multiplexed local-area networks. Fibre and Integrated Optics 1997; 16(3):237-260.

10. El-Bawab TS, Jayasumana AP. Modeling and performance analysis of a symmetric fast-circuit switched robustWDM LAN with the AR/LTP protocol. IEEE Journal of Lightwave Technology 1999; 17(6):973-988.

11. El-Bawab TS, Jayasumana AP. On the design of robust-WDM local-area networks. International Journal of Communication Systems 2001; 14: 231-241.

12. El-Bawab TS, Jayasumana AP. Internetworking strategies for robust-WDM local-area networks. Proceedings of the SPIE International Symposium on Voice, Video and Data Communications, (Broadband Networks-Internet II: Quality of Service and Future Directions), Boston, MA, September 1999.

13. El-Bawab TS, Vaishnav C, Jayasumana AP, Temkin H, Sauer J, Willebrand H. Medium access control protocols for robust WDM local-area networks. Proceedings of the International Conference on Communications (ICC), June 1996. Dallas, TX, pp. 1096-1106.

14. Sauer J, Jayasumana AP, Radehaus C, Temkin H. A robust WDM communication system. Proceedings of the IEEE lasers and Electro-Optics Society 7th Annual Meeting (LEOS'94), October 1994. Boston, MA.

15. Willebrand H, Sauer J, Radehaus C, Sprenger M, Kalra P, Jayasumana AP, Temkin H. A Wavelength tolerant robust optical WDM network based on an intelligent multichannel wavelength tracking receiver. Proceedings of the 2nd International Workshop on High-Speed Network Computing (HiNet96), April 1996. Honolulu, Hawaii.

16. Willebrand H, Sauer J. Adaptive robust optical fibre receiver/transmitter. Proceedings of SPIE, San Jose, CA, January 29-31, 1995; 40-49.

17. Temkin H, Jayasumana AP, Sauer J. Wavelength tolerant WDM networks. SPIE Proceedings, vol. 2524 (NSF Forum on Optical Science and Engineering), July 1995. San Diego, CA, pp. 84-87.

18. Vaishnav C, Nieberger M, Jayasumana AP, Sauer J. Design and performance of a robust WDM network. Current Developments in Optical Design and Engineering VI, vol. 2863, 5-7 August 1996; 370-378.

19. Cooper RB. Queues served in cyclic order: waiting times. The Bell System Technical Journal 1970; 49:399-413.

20. Fuhrmann SW. Symmetric queues served in cyclic order. Operations Research Letters 1985; 4(3):139-144.

21. Vaishnav C. Event driven simulation and performance evaluation of robust WDM networks, M.S. Thesis, Department of Electrical Engineering, Colorado State University, 1997.

\section{AUTHORS' BIOGRAPHIES}

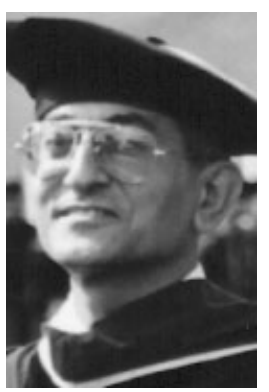

Samir A. Abd-Elmalak received the BS degree in electrical engineering from the Military Technical College, Cairo, Egypt, in 1977, MS degree in Computer Engineering from Cairo University, Egypt in 1989, and the $\mathrm{PhD}$ in Electrical Engineering from Colorado State University in 1997. He also has a diploma in Computer Science from Norsk Data Corp., Norway. He is currently with the Egyptian Military.

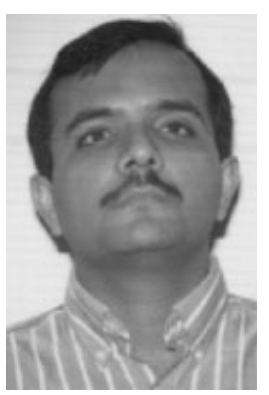

Chintan Vaishnav is a member of technical staff at Avaya Labs in Denver, Colorado. His current responsibility is architecture of system management infrastructure for converged networks. This work focuses on reliability, installability, maintainability, usability, and serviceability issues of converged products. Chintan joined Bell Labs, Lucent Technologies after his MS in Electrical Engineering from Colorado State University in Fort Collins, Colorado. During his masters degree he worked on simulation of Wavelength Division Multiplexed (WDM) networks. Chintan also holds a BE in Electronics Engineering from Bangalore University, India. 


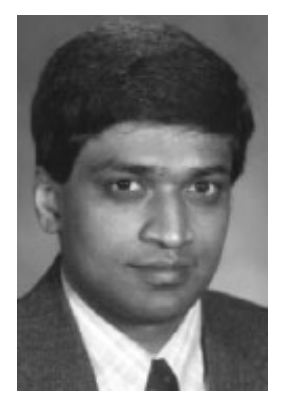

Anura P. Jayasumana is a Professor in the Electrical and Computer Engineering Department, and the Computer Science Department at the Colorado State University. He received his $\mathrm{PhD}$ in 1985 and MS in 1982, both in electrical engineering from the Michigan State University, and the BSc degree in electronics and telecommunication engineering, with first class honors, from the University of Sri Lanka, Moratuwa, in 1978. His research interests include high-speed network protocols, performance evaluation, and VLSI testing. He has authored/co-authored over 130 publications in these areas and a book 'FDDI and FDDI-II: Architecture, Protocols and Performance.' He has served extensively as a consultant to industry. Dr Jayasumana is a senior member of the IEEE, and a member of Phi Kappa Phi, the IEEE Computer Society, and the IEEE Communications Society. He was the winner of the award for the best student in electrical engineering at the University of Sri Lanka, Moratuwa in 1978, and the College of Engineering Outstanding Academic Achievement Awards at Michigan State University in 1982 and 1983. He received the Outstanding Faculty of the Year Award from the Mountain States Council of the American Electronics Association in 1990, and Engineering Deans Council Award for Academic Excellence from the Colorado State University in 1998. 